\title{
MECHANISMS FOR HOUSE ALLOCATION WITH EXISTING TENANTS UNDER DICHOTOMOUS PREFERENCES
}

\author{
Haris Aziz \\ Data61, CSIRO and UNSW Sydney, Australia \\ haris.aziz@data61.csiro.au
}

\begin{abstract}
We consider house allocation with existing tenants in which each agent has dichotomous preferences. We present strategyproof, polynomial-time, and (strongly) individually rational algorithms that satisfy the maximum number of agents. For the endowment only model, one of the algorithms also returns a core-stable allocation.
\end{abstract}

Keywords: House allocation, core, dichotomous preferences.

JEL Classification Numbers: C62, C63, C78.

\section{INTRODUCTION}

Principled allocation of resources is a central problem faced by society. We consider an allocation setting in which there is a set of agents $N=\{1, \ldots, n\}$, a set of houses $H=\left\{h_{1}, \ldots, h_{m}\right\}$, each agent owns at most one and possibly zero houses from $H$ and there may be houses that are not owned by any agent. In the literature the setting is termed as house allocation with existing tenants (Abdulkadiroğlu \& Sönmez, 1999; Sönmez \& Ünver, 2010). If each agent owns exactly one house and each house is owned by some agent, the setting is equivalent to the housing market setting (D. J. Abraham et al., 2005; Aziz \& de Keijzer, 2012; Ma, 1994; Plaxton, 2013; Shapley \& Scarf, 1974).

The author thanks the reviewers for helpful feedback which helped improve the presentation. The author also thanks Péter Biró, Greg Plaxton, Zhaohong Sun, and the anonymous reviewers for useful comments and pointers. 
If no agent owns a house, the setting is equivalent to the house allocation model (Hylland \& Zeckhauser, 1979; Svensson, 1994).

The setting captures various allocations settings including allocation of dormitory rooms as well as kidney exchange markets. We consider housing market with existing tenants setting with the assumption that each agent has dichotomous preferences with utility zero or one.

Dichotomous preferences are prevalent in many settings. A kidney may either be compatible or incompatible for a patient. A dormitory room may be within budget or outside budget for a student. Bogomolnaia \& Moulin (2004) give numerous other examples where dichotomous preferences make sense in allocation and matching problems. Interestingly, since dichotomous preferences require indifference in preferences, they are not covered by many models considered in the literature that assume strict preferences (Abdulkadiroğlu \& Sönmez, 1999; Sönmez \& Ünver, 2010). If a house is acceptable, we will refer to it as an acceptable house. If an agent gets a house that is acceptable, we will say that the agent is satisfied.

For the setting, we study how to allocate the houses to the agents in a desirable manner. There are several properties that capture how to allocate resources in a desirable manner. We consider the standard normative properties in market design: (i) Pareto optimality: there should be no other allocation in which each agent is at least as happy and at least one agent is strictly happier (ii) individual rationality (IR): no agent should have an incentive to leave the allocation program (iii) strategyproofness: no agent should have an incentive to misreport his preferences. We also consider a stronger notion of individual rationality that requires that either an agent keeps his endowment or gets some house that is strictly better. Finally, we consider a property stronger than Pareto optimality: maximizing the number of agents who are satisfied. If we rephrase dichotomous preferences in terms of 1-0 utilities, the goal is equivalent to maximizing social welfare. Under 1-0 utilities, an allocation maximizes welfare if a maximum number of agents get utility 1 or in other words a maximum number of agents are satisfied. Throughout the paper, when we refer to (utilitarian) welfare, we will assume that the underlying cardinal utilities are 1-0.

The main results are as follows:

Theorem 1. For house allocation with existing tenants with dichotomous preferences, there exists an algorithm (MSIR) that is polynomial-time, strategyproof, and allocates acceptable houses to a maximum number of agents 
subject to strong individual rationality. In the endowment setting in which each house is initially owned by an agent, the algorithm is core stable.

Theorem 2. For house allocation with existing tenants with dichotomous preferences, there exists an algorithm (MIR) that is polynomial-time, strategyproof, allocates acceptable houses to a maximum number of agents, and is Pareto optimal.

\section{RELATED WORK}

House allocation with existing tenants model was introduced by Abdulkadiroğlu \& Sönmez (1999). They assumed that agents have strict preferences and introduced mechanisms that are individually rational, Pareto optimal and strategyproof. The results do not apply to the setting with dichotomous preferences.

In the restricted model of housing markets, Jaramillo \& Manjunath (2012) proposed a mechanism called TCR that is polynomial-time, IR (but not S-IR), Pareto optimal and strategyproof even if agents have indifferences. The results also apply to housing markets under dichotomous preferences. The MIR and MSIR mechanisms are relatively simpler and the arguments for their properties are simple and direct as well.

Another natural approach to allocation problems is to satisfy the maximum number of agents by computing a maximum weight matching. However, such an approach violates individual rationality. Krysta et al. (2014) proposed algorithms for house allocation with 1-0 utility. However their algorithms are for the model in which agents do not have any endowment.

For kidney exchange with 1-0 utilities, D. Abraham et al. (2007) and Biro et al. (2009) presented an algorithm that is strongly individually rational and satisfies the maximum number of agents. The algorithm does not cater for the cases where agents own acceptable houses, or there may be houses that are not owned by any agents. Furthermore, they did not establish strategyproofness or core stability of their mechanism.

\section{PRELIMINARIES}

Let $N=\{1, \ldots, n\}$ be a set of $n$ agents and $H=\left\{h_{1}, \ldots, h_{m}\right\}$ a set of $m$ houses. The endowment function $e: N \rightarrow H \cup\{$ null $\}$ assigns to each agent the house he originally owns. The value $e(i)$ is null if agent $i$ does not own a house. 
Each agent has complete and transitive preferences $\succsim_{i}$ over the houses and $\succsim\left(\succsim_{1}, \ldots \succsim_{n}\right)$ is the preference profile of the agents. The housing market is a quadruple $M=(N, H, e, \succsim)$. For $S \subseteq N$, we denote $\bigcup_{i \in S}\{e(i)\}$ by $e(S)$. A function $x: S \rightarrow H$ is an allocation on $S \subseteq N$ tha allocates house $x(i)$ to agent $i \in N$. The goal is to allocate the houses in a mutually beneficial and efficient way.

Since we consider dichotomous preferences, we will denote by $A_{i}$ the set of houses acceptable by $i \in N$. These houses are acceptable to $i$ whereas the other houses are unacceptable to $i$. Agent $i$ is satisfied when he gets an acceptable house. Since we consider welfare as well, we will assume that acceptable houses give utility one to the agent and unacceptable houses give utility zero. Hence getting an unacceptable house is equivalent to not getting a house.

An allocation $x$ is individually rational $(I R)$ if $x(i) \succsim_{i} e(i)$ for all $i \in N$. IR requires that an agent endowed with an acceptable house is allocated an acceptable house. An allocation $x$ is strongly individually rational $(S-I R)$ if $x(i)=e(i)$ or $x(i) \succ_{i}(e(i))$ for all $i \in N$. SIR requires that an agent only changes his house if he gets a strictly more preferred house.

A coalition $S \subseteq N$ blocks an allocation $x$ on $N$ if there exists an allocation $y$ on $S$ such that for all $i \in S, y(i) \in e(S)$ and $y(i) \succ_{i} x(i)$. An allocation $x$ on $N$ is in the core $(C)$ of market $M$ if it admits no blocking coalition. An allocation that is in the core is also said to be core stable. A coalition $S \subseteq N$ weakly blocks an allocation $x$ on $N$ if there exists an allocation $y$ on $S$ such that for all $i \in S, y(i) \in e(S), y(i) \succsim_{i} x(i)$, and there exists an $i \in S$ such that $y(i) \succ_{i} x(i)$. An allocation $x$ on $N$ is in the strict core $(S C)$ of market $M$ if it admits no weakly blocking coalition. An allocation that is in the strict core is also said to be strict core stable. An allocation is Pareto optimal (PO) if $N$ is not a weakly blocking coalition. It is clear that strict core implies core and also Pareto optimality. Core implies individual rationality.

From now on we will assume 1-0 utilities for all the statements.

Example 1. Consider a house allocation with existing tenants setting:

- $N=\{1,2,3,4,5\}$;

- $H=\left\{h_{1}, h_{2}, h_{3}, h_{4}\right\}$;

- $e(i)=h_{i}$ for $i \in\{1, \ldots, 4\} ; e(5)=$ null

- $A_{1}=\left\{h_{2}\right\} ; A_{2}=\left\{h_{3}\right\} ; A_{3}=\left\{h_{1}\right\}, A_{4}=\left\{h_{3}\right\}, A_{5}=\left\{h_{4}\right\}$ 
A feasible S-IR outcome is allocation $x$ such that $x(1)=h_{2}, x(2)=h_{3}, x(3)=$ $h_{1}, x(4)=h_{4}$, and $x(5)=$ null.

A feasible IR and Pareto optimal outcome is allocation y such that $y(1)=$ $h_{2}, y(2)=h_{3}, y(3)=h_{1}, y(4)=n u l l$, and $y(5)=h_{4}$.

It is obvious that S-IR implies IR. We also observe the following.

Proposition 1. An allocation that maximizes welfare subject to S-IR may have less welfare than an allocation that maximizes welfare subject to IR.

Proof. Consider the setting in which there are two agents and both agents have zero value houses but one agent wants the other agent's house. The only feasible S-IR allocation is the endowment allocation whereas there exists an IR allocation in which one agents gets an acceptable.

\section{MECHANISMS}

We present two mechanisms: MSIR and MIR. MSIR satisfies the maximum number of agents subject to the S-IR constraint. MIR satisfies the maximum number of agents subject to the IR constraint. Both mechanisms are based on constructing a bipartite graph that admits a perfect matching and repeatedly modifying the graph while still ensuring that the graph still has a maximum weight perfect matching. The mechanisms are parametrized by a permutation $\pi$ that is a function $\pi: N \rightarrow N$. The function specifies an ordering of the agents in $N$ so that $\pi(j)$ is the $j$-th agent in the ordering.

\subsection{The MSIR Mechanism}

The MSIR mechanism is specified as Algorithm 1.

Example 2. Let us illustrate how the MSIR mechanism works on the setting in Example 1. Suppose $\pi=12345$. First we form a graph in Figure 1. We find a perfect matching that satisfies the most number of agents subject to $S$-IR. Such a matching can satisfy at most 3 agents. We go through permutation 12345 and check whether there exists a matching that satisfies 3 agents, does not violate $S$-IR, satisfied the guarantees of previous agents in the permutation, and still satisfies the current agent in the permutation. If yes, that agent is guaranteed to be satisfied from now. After agents 1, 2, and 3 are processed, we know that 1 should get $h_{2}, 2$ should get $h_{3}$ and 3 should get $h_{1}$. S-IR requires that 4 and 


\section{Algorithm 1 MSIR}

Input: $\quad\left(N, H,\left(A_{1}, \ldots, A_{n}\right), e\right)$

Output: Allocation $(x(1), \ldots, x(n))$

1 Consider weighted graph $G=(A \cup B, E, w)$. Set $k$ to $|n-m|$. If $m>n$, then $A=N \cup D_{N}$ where $D_{N}=\left\{d_{1}, \ldots, d_{k}\right\}$. If $n>m$, then $B=H \cup D_{H}$ where $D_{H}=\left\{o_{1}, \ldots, o_{k}\right\}$. If $n=m$, then $A=N$ and $B=H$.

$E$ is defined as follows:

- For each $a \in N$ and $b=e(a)$, add $\{a, b\} \in E$ with $w(\{a, b\})=0$ if $b \notin A_{a}$ and $w(\{a, b\})=1$ if $b \in A_{a}$.

- For each $a \in N$ such that $e(a) \neq$ null, $e(a) \notin A_{a}$, and each $b=H \backslash\{e(a)\}$ such that $b \in A_{a}$, add $\{a, b\} \in E$ with $w(\{a, b\})=1$.

- For each $a \in N$ such that $e(a)=$ null and each $b \in B$, add $\{a, b\} \in E$ with $w(\{a, b\})=0$ if $b \notin A_{a}$ and $w(\{a, b\})=1$ if $b \in A_{a}$.

- For each $b \in D_{H}$ and each $a \in\{a \in N: e(a)=n u l l\}$, add $\{a, b\} \in E$ with $w(\{a, b\})=0$.

- For each $a \in D_{N}$ and each $b \in B$, add $\{a, b\} \in E$ with $w(\{a, b\})=0$.

\{Note that if an agent has an acceptable endowment, then he only has an edge to his endowment. An agent with an unacceptable endowment only has edges with his endowment as well as the acceptable houses.

2 Compute a maximum weight perfect matching of $G$. Let the weight of the matching be $W$.

3 Take a permutation (ordering) $\pi$ of the elements in $N$. Denote by $\pi(i)$ the $i$-th element in the ordering.

4 for $i=1$ to $n$ do

5 Set $t_{\pi(i)}$ to 1.

6 Remove from $G$ each edge $\{\pi(i), b\} \in E$ such that $w(\{\pi(i), b)\})=0$. Compute a maximum weight perfect matching of $G$. If the weight is less than $W$ or if there does not exist a perfect matching, then put back the removed edges and set $t_{\pi(i)}$ to 0 . $\left\{\right.$ Variable $t_{\pi(i)}$ keeps track of whether agent $\pi(i)$ should get an acceptable house or not.

\section{7 end for}

8 Compute a maximum weight perfect matching $M$ of $G$. Consider the allocation $x$ in which each agent $i \in N$ gets a house that it is matched to in $M$. If $i \in N$ is matched to a dummy house, its allocation is null.

9 return $(x(1), \ldots, x(n))$

5 do not get any new house. Hence the result is the same as allocation $x$ in Example 1. 


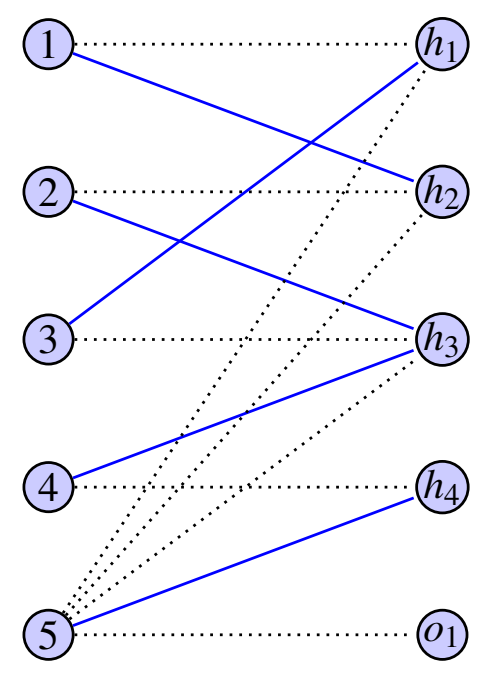

Figure 1: Initial graph $G$ when running MSIR over the instance in Example 1. The solid edges have weight 1 whereas the dotted edges have weight 0 . A perfect matching with the maximum weight has weight 3 .

\section{Proposition 2. MSIR runs in polynomial-time.}

Proof. The graph $G$ has $O(n+m)$ vertices. The graph is modified at most $O(n)$ times. Each time, a maximum weight perfect matching is computed that takes polynomial time $|V(G)|^{3}$ (Korte \& Vygen, 1999).

Proposition 3. MSIR returns an allocation that is S-IR.

Proof. Throughout the algorithm, we make sure that $G$ admits a perfect matching. If a modification to $G$ leads to a lack of a perfect matching, then such a modification is reversed. An agent with an endowment only has an edge to his endowment or to an acceptable house. Therefore while a perfect matching exists, an agent with a non-null endowment cannot be matched to an unacceptable house other than his endowment. Thus MIR returns an allocation that is S-IR.

Proposition 4. MSIR returns an allocation that satisfies the maximum number of agents subject to $S$-IR.

Proof. Throughout the algorithm, we make sure that $G$ admits a perfect matching which ensures that the corresponding allocation satisfies S-IR. An agent 
with an acceptable endowment does not have an edge to any other house so he has to be allocated his endowment. An agent with an unacceptable endowment either has an edge to his endowment and to houses that are acceptable. Hence, in a perfect matching, the agent either gets his endowment or an acceptable house. Given this condition, we compute the maximum weight perfect matching. This implies that the corresponding allocation satisfies the maximum number of agents under the S-IR constraint.

Corollary 1. MSIR returns an allocation that is Pareto optimal among the set of $S$-IR allocations.

Proof. Assume for contradiction that the MSIR allocation is Pareto dominated by an S-IR allocation. But this means that the MSIR allocation does not satisfy the maximum number of agents.

Proposition 5. MSIR is strategyproof.

Proof. MSIR returns a perfect matching of weight $W$. During the running of MSIR, each time a modification is made to the graph $G$, it is ensured that $G$ admits a perfect matching of weight $W$. Assume for contradiction that MSIR is not strategyproof and some agent $i \in N$ with turn $k$ in permutation $\pi$ gets a more preferred house when he misreports. This means that agent $i$ gets an unacceptable house when he reports the truthful preference $A_{i}$. Let the allocation be $x$. This implies that in permutation $\pi$, when $i$ 's turn comes, there exists no feasible maximum weight perfect matching of weight $W$ in which $i$ gets an acceptable house and each agent $\pi(j)$ preceding $i$ in permutation $\pi$ gets $t_{\pi(j)}$ acceptable houses. Since $i$ can get a more preferred house by misreporting, $i$ gets an acceptable house if he reports $A_{i}^{\prime}$. Let such an allocation be $x^{\prime}$. Note that $x^{\prime}$ is a feasible maximum weight perfect matching even when $i$ tells the truth and even if each agent $\pi(j)$ preceding $i$ in permutation $\pi$ gets $t_{\pi(j)}$ acceptable houses. But this is a contradiction because there does exist a feasible maximum weight perfect matching of weight $W$ in which $i$ gets an acceptable house and each agent preceding $i$ in permutation $\pi$ gets $t_{\pi(j)}$ acceptable houses.

Lemma 1. For 1-0 utilities, any S-IR welfare maximizing allocation is core stable.

Proof. Suppose that there is a blocking coalition $S$. It can only consist of agents who did not get an acceptable house in the allocation. S-IR implies that 
agents who originally own an acceptable house keep the acceptable house. If an agent $i \in N$ is in $S$ who does not originally own any house, he cannot be part of $S$ because he has nothing to give to other agents, so other agents can satisfy each other without letting $i$ be a member of $S$. Therefore, $S$ consists of those agents who owned an unacceptable house and are allocated an unacceptable house. Due to S-IR, agents in $S$ are allocated their own house. Now if $S$ admits a blocking coalition this implies that the S-IR welfare maximizing allocation was not S-IR welfare maximizing which is a contradiction.

In view of the lemma, we derive the following statement.

Proposition 6. MSIR returns an allocation that is core stable.

\subsection{The MIR Mechanism}

The MIR mechanism is specified as Algorithm 2. The main difference between MIR and MSIR is that we impose the less restrictive IR constraint for MIR rather than the S-IR constraint. In MIR, an agent who owns an acceptable house is willing to get some other acceptable house. If the agent owns an unacceptable house, he is willing to give that house to someone else even if he does not get an acceptable house in return.

Example 3. Let us illustrate how the MIR mechanism works on the setting in Example 1. Suppose $\pi=12345$. First we form a graph in Figure 2. We then find a perfect matching that satisfies the most number of agents subject to IR. Such a matching can satisfy at most 4 agents. We go through permutation 12345 and check whether there exists a matching that satisfies 4 agents, does not violate IR, satisfies the guarantees of the previous agents in the permutation, and still satisfies the current agent in the permutation. If yes, we require that agent to be satisfied. After agents 1, 2, and 3 are processed, we know that 1,2 , and 3 can be satisfied by giving them $h_{2}, h_{3}$, and $h_{1}$ respectively. Agent 4 cannot be satisfied while requiring agents 1, 2, and 3 to be satisfied. The last agent 5 can be satisfied without violating IR or resulting in some agent among 1, 2, and 3 to be not satisfied. The outcome is the same as allocation $y$ in Example 1.

Proposition 7. MIR runs in polynomial-time. 


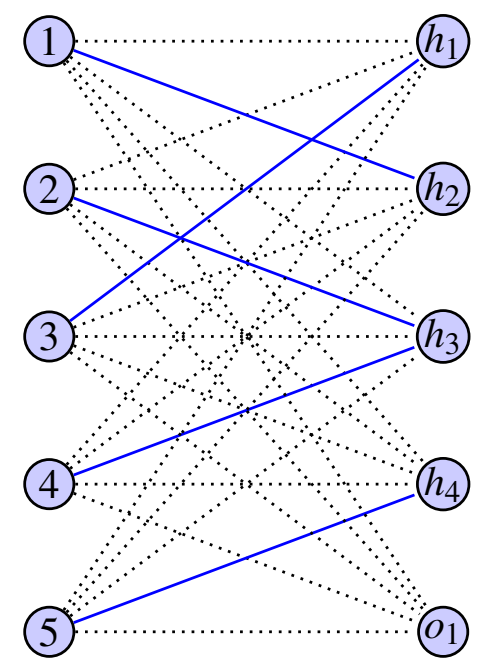

Figure 2: Initial graph $G$ when running MIR over the instance in Example 1. The solid edges have weight 1 whereas the dotted edges have weight 0 . A perfect matching with the maximum weight has weight 4 .

Proof. The graph $G$ has $O(n+m)$ vertices. The graph is modified at most $O(n)$ times. Each time, a maximum weight perfect matching is computed that takes time $|V(G)|^{3}$ (Korte \& Vygen, 1999).

Proposition 8. MIR returns an allocation that is $I R$.

Proof. Throughout the algorithm, we make sure that $G$ admits a perfect matching. If a modification to $G$ leads to a lack of a perfect matching, then such a modification is reversed. An agent with an acceptable endowment only has an edge to his endowment or to other acceptable houses. Therefore while a perfect matching exists, an agent with an acceptable endowment can only be matched to an acceptable house. Thus MIR returns an allocation that is IR.

Proposition 9. MIR returns an allocation that satisfies the maximum number of agents subject to IR.

Proof. Throughout the algorithm, we make sure that $G$ admits a perfect matching which ensures that the corresponding allocation satisfies IR. Given this condition, we compute the maximum weight perfect matching. This implies that the corresponding allocation satisfies the maximum number of agents under the IR constraint. 


\section{Algorithm 2 MIR}

Input: $\quad\left(N, H,\left(A_{1}, \ldots, A_{n}\right), e\right)$

Output: $(x(1), \ldots, x(n))$

1 Consider weighted graph $G=(A \cup B, E, w)$. Set $k$ to $|n-m|$. If $m>n$, then $A=N \cup D_{N}$ where $D_{N}=\left\{d_{1}, \ldots, d_{k}\right\}$. If $n>m$, then $B=H \cup D_{H}$ where $D_{H}=\left\{o_{1}, \ldots, o_{k}\right\}$. If $n=m$, then $A=N$ and $B=H$.

$E$ is defined as follows:

- For each $a \in N$ and $b=e(a)$, add $\{a, b\} \in E$ with $w(\{a, b\})=0$ if $b \notin A_{a}$ and $w(\{a, b\})=1$ if $b \in A_{a}$.

- For each $a \in N$ and each $b \in H \backslash\{e(a)\}$ such that $b \in A_{a}$, add $\{a, b\} \in E$ with $w(\{a, b\})=1$.

- For each $a \in N$ such that $e(a)=$ null and each $b \in B$, add $\{a, b\} \in E$ with $w(\{a, b\})=0$ if $b \notin A_{a}$ and $w(\{a, b\})=1$ if $b \in A_{a}$.

- For each $a \in N$ such that $e(a)=h$ and $h \notin A_{a}$ and each $b \in B$, add $\{a, b\} \in E$ with $w(\{a, b\})=0$ if $b \notin A_{a}$ and $w(\{a, b\})=1$ if $b \in A_{a}$.

- For each $b \in D_{H}$ and each $a \in\left\{a \in N: e(a) \notin A_{a}\right\}$, add $\{a, b\} \in E$ with $w(\{a, b\})=0$.

- For each $a \in D_{N}$ and each $b \in B$, add $\{a, b\} \in E$ with $w(\{a, b\})=0$.

\{Note that if an agent has an acceptable endowment, he only has edges with acceptable houses. An agent with an unacceptable endowment has an edge to every $b \in B$. $\}$

2 Compute a maximum weight perfect matching of $G$. Let the weight of the matching be $W$.

3 Take a permutation (ordering) $\pi$ of the elements in $N$. Denote by $\pi(i)$ the $i$-th element in the ordering.

4 for $i=1$ to $n$ do

Set $t_{\pi(i)}$ to 1 .

6 Remove from $G$ each edge $\{\pi(i), b\} \in E$ such that $w(\{\pi(i), b)\})=0$. Compute the maximum weight perfect matching of $G$. If the weight is less than $W$ or if there does not exist a perfect matching, then put back the removed edges and set $t_{\pi(i)}$ to 0 . $\left\{\right.$ Variable $t_{\pi(i)}$ keeps track of whether agent $\pi(i)$ should get an acceptable house or not. $\}$

7 end for

8 Compute a maximum weight perfect matching $M$ of $G$. Consider the allocation $x$ in which each agent $i \in N$ gets a house that it is matched to in $M$. If $i \in N$ is matched to a dummy house, its allocation is null.

9 return $(x(1), \ldots, x(n))$ 
Lemma 2. An allocation that maximizes welfare subject to IR has the same welfare even if IR is not imposed.

Proof. A matching is maximum size if and only if there exist no improving path, which is an alternating path starting from an unmatched agent and ending with an unallocated house (König's theorem (see e.g., Lovász \& Plummer, 2009)). Therefore if the obtained individually rational matching, $M$, was not maximum size then we could improve its size in such a way that all matched agents in $M$ remain matched, contradicting with the maximality of $M$ among IR matchings.

As a result of the lemma, we obtain the following proposition.

Proposition 10. MIR returns an allocation that satisfies the maximum number of agents.

Corollary 2. MIR returns an allocation that is Pareto optimal.

The argument for MIR being strategyproof is exactly the same as that of MSIR being strategyproof.

Proposition 11. MIR is strategyproof.

For housing markets, in contrast to MSIR, MIR may not be core stable.

Proposition 12. For 1-0 utilities, an IR welfare maximizing allocation may not be core stable.

Proof. Consider a four agent setting in which each agent owns a house unacceptable to himself. Agent 2 owns a house that is acceptable to 4 and 1 and agent 1 owns a house that is acceptable to 2 and 3 . Consider an allocation in which agent 3 gets 1's house and 4 gets 2's house. Such an allocation is IR and satisfies the maximum number of agents. However, it is not core stable because 1 and 2 can form a blocking coalition.

In this paper, two new mechanisms called MSIR and MIR were introduced. See Table 1 for a summary of properties satisfied by the mechanisms for house allocation with existing tenants. 


\begin{tabular}{lcc}
\hline & MSIR & MIR \\
\hline Strategyproof & + & + \\
S-IR & + & - \\
IR & + & + \\
Core stable & + & - \\
Pareto optimal & - & + \\
Max welfare subject to S-IR & + & - \\
Max welfare subject to IR & - & + \\
Max welfare & - & + \\
\hline
\end{tabular}

Table 1: Properties satisfied by mechanisms for house allocation with existing tenants with dichotomous preferences.

\section{References}

Abdulkadiroğlu, A., \& Sönmez, T. (1999). House allocation with existing tenants. Journal of Economic Theory, 88(2), 233-60.

Abraham, D., Blum, A., \& Sandholm, T. (2007). Clearing algorithms for barter exchange markets: Enabling nationwide kidney exchanges. In: Proceedings of the 8th ACM Conference on Electronic Commerce (ACM-EC), ACM Press, 295-304.

Abraham, D. J., Cechlárová, K., Manlove, D. F., \& Mehlhorn, K. (2005). Pareto optimality in house allocation problems. In: Proceedings of the 16th International Symposium on Algorithms and Computation (ISAAC), Vol. 3341 of Lecture Notes in Computer Science (LNCS), 1163-75.

Aziz, H., \& de Keijzer, B. (2012). Housing markets with indifferences: A tale of two mechanisms. In: Proceedings of the 26th AAAI Conference on Artificial Intelligence (AAAI), 1249-55.

Biro, P., Manlove, D. F., \& Rizzi, R. (2009). Maximum weight cycle packing in optimal kidney exchange programs. Discrete Mathematics, Algorithms and Applications, 1(4), 499-517.

Bogomolnaia, A., \& Moulin, H. (2004). Random matching under dichotomous preferences. Econometrica, 72(1), 257-79.

Hylland, A., \& Zeckhauser, R. (1979). The efficient allocation of individuals to positions. Journal of Political Econom, 87(2), 293-314.

Jaramillo, P., \& Manjunath, V. (2012). The difference indifference makes in strategyproof allocation of objects. Journal of Economic Theory, 147(5), 1913-46.

Korte, B., \& Vygen, J. (1999). Combinatorial optimization (5th ed.). Heidelberg, Germany: Springer-Verlag. 
Krysta, P., Manlove, D. F., Rastegari, B., \& Zhang, J. (2014). Size versus truthfulness in the house allocation problem. In: Proceedings of the 15th ACM Conference on Economics and Computation (ACM-EC), ACM Press, 453-70.

Lovász, L., \& Plummer, M. D. (2009). Matching theory. AMS Chelsea Publishing. Ma, J. (1994). Strategy-proofness and the strict core in a market with indivisibilities. International Journal of Game Theory, 23(1), 75-83.

Plaxton, C. G. (2013). A simple family of top trading cycles mechanisms for housing markets with indifferences. In: Proceedings of the 24th International Conference on Game Theory.

Shapley, L. S., \& Scarf, H. (1974). On cores and indivisibility. Journal of Mathematical Economics, 1(1), 23-37.

Sönmez, T., \& Ünver, M. U. (2010). House allocation with existing tenants: A characterization. Games and Economic Behavior, 69(2), 425-45.

Svensson, L.-G. (1994). Queue allocation of indivisible goods. Social Choice and Welfare, 11, 323-30. 